\title{
DAB2 suppresses gastric cancer migration by regulating the Wnt/ $\beta$-catenin and Hippo-YAP signaling pathways
}

\author{
Hua Wang ${ }^{1 \#}$, Surong Dong ${ }^{2 \#}$, Yun Liu ${ }^{1}$, Feng Ma ${ }^{3}$, Jian Fang ${ }^{1}$, Wentao Zhang ${ }^{1}$, Shihe Shao ${ }^{1}$, Hongxing Shen ${ }^{1}$, \\ Jingpeng Jin $^{3}$
}

${ }^{1}$ School of Medicine, Jiangsu University, Zhenjiang 212013, China; ${ }^{2}$ Department of Laboratory Medicine, Taizhou People's Hospital, Taizhou 225300, China; ${ }^{3}$ Department of Endoscopy Center, China-Japan Union Hospital of Jilin University, Changchun 130033, China

Contributions: (I) Conception and design: H Wang, J Jin, H Shen; (II) Administrative support: S Shao, W Zhang; (III) Provision of study materials or patients: None; (IV) Collection and assembly of data: J Fang; (V) Data analysis and interpretation: S Dong, Y Liu; (VI) Manuscript writing: All authors; (VII) Final approval of manuscript: All authors.

\#These authors contributed equally to this work.

Correspondence to: Jingpeng Jin. Department of Endoscopy Center, China-Japan union hospital of Jilin University, 126 Xiantai Street, Changchun 130033, China. Email: jpjin@sohu.com; Hongxing Shen. School of Medicine, Jiangsu University, 301 Xuefu Road, Zhenjiang 212013, China. Email: hxshen@ujs.edu.cn.

Background: Disabled-2 (DAB2), a potential tumor suppressor, plays an in important role in cancer development and cellular differentiation. Its lower expression levels have founded in many cancers. In addition, DAB2 is involved in multiple signaling pathways, including TGF- $\beta$ and Wnt signal pathways. Gastric cancer (GC) is a common gastrointestinal malignant tumor. Nonetheless, the role of DAB2 in GC remains unclear.

Methods: Thirty-seven clinical specimens of GC tissues and adjacent non-tumor tissues were examined by immunohistochemistry. Proteins were extracted from two of them to perform Western blot analysis. Then, CMV-MCS-3FLAG-SV40-DAB2 and si-DAB2 were transfected into MGC and SGC cell line, respectively. The migration of GC cells was evaluated by transwell migration assay. And, the expression of migration related proteins was detected by Western blot and immunofluorescence (IF).

Results: Eighty-six percent (32/37) of patients DAB2 staining was reduced in GC tissues compared to adjacent normal tissues. Further studies showed that in six human GC cell lines, the level of DAB2 expression was lower than normal gastric epithelial cells, and that DAB2 was closely related to cell migration in vitro. In DAB2 silenced cells, the $\mathrm{Wnt} / \beta$-catenin signaling was increased and the Hippo-YAP pathway was affected. In addition, lower DAB2 level led to nuclear translocation of $\beta$-catenin and Yap.

Conclusions: The lower expression of DAB2 regulates cell migration in GC via interfering with the Wnt and Hippo signaling pathway. Our findings suggested that DAB2 played an important role in the migration of GC.

Keywords: Gastric cancer (GC); disabled-2 (DAB2); migration; Wnt//-catenin; Hippo-YAP

Submitted Sep 11, 2019. Accepted for publication Dec 17, 2019.

doi: $10.21037 /$ tcr.2019.12.96

View this article at: http://dx.doi.org/10.21037/tcr.2019.12.96

\section{Introduction}

Gastric cancer (GC) is the fifth most common cancer in the world after lung, breast, prostate, and colorectal cancers (1). Over the past decades, various therapeutic options have been improving, including surgical resection, chemotherapy and radiotherapy (2). Nevertheless, about $5 \%$ to $30 \%$ of GC patients develop recurrent disease at stage I, and $60 \%$ of patients have locally advanced disease, finally dying of metastatic disease (3). Therefore, more therapeutic strategies and molecular mechanisms against 
GC development and progression are needed, especially against invasion and metastasis.

The disabled-2 (DAB2) gene expressed in many types of epithelial cells has an important impact on various functions, such as endocytosis, cytoskeletal reorganization and cell adhesion, and so on (4-6). It is also shown to play crucial roles in cancer development and cellular differentiation. Moreover, in the metastases of colorectal cancer, prostate and bladder cancers, the expression of DAB2 is down-regulated (6-8). As a potential tumor suppressor, DAB2 is involved in multiple signaling pathways. In pancreatic ductal adenocarcinoma, DAB2 expression promotes EMT by modulating TGF- $\beta$ responses (9). DAB2 suppresses the Wnt signal pathway by preventing PP1 (protein phosphatase) (10). In addition, by regulating the endocytic fate of the lipoprotein receptor-related protein 6 receptor, DAB2 participates in the regulation of the Wnt signal pathway (11). In spite of these findings, further studies are essential for gaining new insights into the relationship between DAB2 and cancer.

Thus, our goal was to delineate the expression and functional aspects of DAB2 in GC. This study disclosed that DAB2 expression was decreased whether in GC tissues or human GC cell lines. Further functional studies showed that $\mathrm{DAB} 2$ was closely related to cell migration in vitro. Moreover, knockdown of DAB2 increased the Wnt/ $\beta$-catenin signaling and affected the Hippo-YAP pathway. Nuclear translocation of Yap and $\beta$-catenin in DAB2silenced cells was also observed. These findings suggested that DAB2, acting as a tumor suppressor, played a critical role in the migration of GC.

\section{Methods}

\section{Tissue specimens and patient clinical information}

In this study, 37 clinical specimens of GC tissues and adjacent non-tumor tissues collected from the ChinaJapan union hospital of Jilin University, Jilin, China, were embedded in paraffin for immunohistochemistry. All the patients had not received treatment before undergoing surgery, and were confirmed by histology to have had GC. Also, proteins were extracted from two of them to perform Western blot analysis. Informed consents were obtained from all the participants enrolled, and the study was approved by institutional ethics committee board of Jiangsu University (No. UJS-IACUC-AP-20190307087).

\section{Cell lines and cultures}

The human GC cell lines (AGS, SGC-7901, MKN-45, BGC-823, MGC-803, HGC) were obtained from our laboratory, whereas the human normal gastric epithelial cell line GES-1 was purchased from the Institute of Biochemistry and Cell Biology, SIBS, CAS (Shanghai, China). The human GC cell line, AGS, was cultured in F12 (Gibco, Grand Island, NY, USA) and the other cell lines were propagated in RPMI 1640 (Gibco, Grand Island, NY, USA). All the media were supplemented with $10 \%$ fetal bovine serum (FBS; Wisent Inc., Quebec, Canada) and cells were cultivated at $37^{\circ} \mathrm{C}$ in a humidified incubator with $5 \% \mathrm{CO}_{2}$.

\section{Plasmid construction, small interference RNA and cell transfection}

A full-length human c-DNA of DAB2 was synthesized and integrated into CMV-MCS-3FLAG-SV40 by Genechem (Shanghai, China), and then transfected into MGC cell line using Lipofectamine 2000 (Invitrogen, Shanghai, China). Moreover, two pairs of oligonucleotides against mRNA areas of DAB2 gene were designed as DAB2 siRNA (forward: CCAGCAGUGAGAACUCAAATT, reverse: UUUGAGUUCUCACUGCUGGTT; GenePharma Corporation, Shanghai, China). Either si-DAB2 or control siRNA used for the downregulation of DAB2, were transfected into the SGC cell line. After culturing for $48 \mathrm{~h}$, the transfected cells were extracted and examined by Western blot to analyze the over-expressed or downregulated efficiency. Then the cells were harvested for further study.

\section{Western blot}

The cells were rinsed with cold PBS and then lysed in RIPA (Radio-Immunoprecipitation Assay) lysis buffer (Beyotime Biotechnology, Shanghai, China) supplemented with PMSF (Phenylmethanesufonyl fluoride) and phosphatase inhibitors. Samples (about $100 \mu \mathrm{g}$ ) were separated via $10 \%$ SDS-PAGE (sodium salt-polyacrylamide gel electrophoresis) and transferred to PVDF (polyvinylidene fluoride member), then blocked in $5 \%$ non-fat milk powder for $1 \mathrm{~h}$ at room temperature and probed at $4{ }^{\circ} \mathrm{C}$ overnight with the following antibodies: anti-DAB2 (1:500, Abcam, UK), anti-GAPDH (glyceraldehyde-3phosphate dehydrogenase), anti-Flag, anti- $\beta$-catenin, anti- 
LATS1 (Large tumor suppressor), anti-p-LATS1, anti-Ecadherin, anti-PCNA (Proliferating cell nuclear antigen), anti-p-GSK $\beta$ (Phosphorylated glycogen synthase kinase 3 $\beta$, 1:1,000, Cell Signaling, Danvers, MA, USA), anti-MMP2 (matrix metalloprotein), anti-MMP9, anti-c-Myc (Cellular myelocytomatosis oncogene), anti-CyclinD1, anti-Yap (Yes associated protein, 1:200, Wanlei, Wanleibio, Liaoning, China), and anti-p-Yap (Phosphorylated Yes associated protein, Ruiying, Ruiyingbio, Suzhou, China). Next, the membranes were incubated with appropriate secondary antibodies (goat anti-rabbit and horse anti-mouse, 1:2,000, Fcmacs, China) for $1 \mathrm{~h}$ at room temperature. The bands were detected using enhanced chemiluminescence (ECL) (Merck Millipore, Darmstadt, Germany) for Western blot analysis. Finally, ImageJ software was used to quantify the relative density of bands.

\section{Immunobistochemistry}

Tissues were fixed with $4 \%$ paraformaldehyde solution, embedded in paraffin and prepared into $4 \mu \mathrm{m}$-thick sections. Then, antigen retrieval was performed by antigen retrieval solution and blocked with 5\% BSA (bovine serum albumin, Boster Bioengineering, Wuhan, China). The tissue sections were incubated with primary antibody against DAB2 (1:100) overnight at $4{ }^{\circ} \mathrm{C}$, then washed and treated with biotinylated secondary antibody. Finally, the sections were visualized with $\mathrm{DAB}$ and counterstained with hematoxylin.

\section{Cell migration assay}

Transfected cells $\left(1 \times 10^{5}\right.$ cell/well $)$ were seeded in the upper chamber of the transwell (Merck Millipore, Darmstadt, Germany) in $200 \mu \mathrm{L}$ serum-free medium, and $600 \mu \mathrm{L}$ medium containing $10 \%$ FBS was added into the lower compartment of the chamber. Following incubation at $37{ }^{\circ} \mathrm{C}$ in $5 \% \mathrm{CO}_{2}$ for $24 \mathrm{~h}$, the cells in the upper surface migrated to the lower surface of the filter. After fixing with $4 \%$ paraformaldehyde, the migrated cells were stained with crystal violet. The images were captured and the invaded cells were counted under a microscope.

\section{Immunofluorescence}

Transfected cells $\left(2.5 \times 10^{4}\right.$ cell/well $)$ were cultured on coverslips in 24-well plates for $24 \mathrm{~h}$. After fixing with $4 \%$ paraformaldehyde, the cells were penetrated with $0.5 \%$ tritonX-100 for $20 \mathrm{~min}$ and blocked in BSA for $30 \mathrm{~min}$ at room temperature, and then incubated with the following antibodies at $4{ }^{\circ} \mathrm{C}$ overnight: anti- $\beta$-catenin (1:75), anti-Yap (1:75), and anti-p-Yap (1:50). After washing with PBS three times, cells were incubated with secondary antibody at $37^{\circ} \mathrm{C}$ for $30 \mathrm{~min}$ and the nuclei were counterstained with Hoechst 33258 (Cell Signaling, Danvers, MA, USA). Images were captured with a confocal microscope (Olympus, USA).

\section{Statistical analysis}

Every experiment was performed at least 3 times and the data were reported as mean $\pm \mathrm{SD}$. Then, the differences between groups were analyzed using one-way ANOVA. All statistical analyses were performed using SPSS19 (SPSS, Chicago, IL, USA) and $\mathrm{P}<0.05$ was considered statistically significant.

\section{Results}

\section{$D A B 2$ expression is decreased in GC tissues and human $G C$ cell lines}

Previous studies have shown that DAB2 expression was decreased in malignant tumors, including breast cancer (12), prostate carcinoma (13), esophageal squamous tumors and so on (7). To explore the role of DAB2 in GC, we first detected the expression of DAB2 in 37 cases of GC patients with paired adjacent non-tumor tissue by immunohistochemical staining. The results indicated that different protein expression levels of DAB2 were detected in GC tissues and the adjacent normal tissues (Figure 1A), and $86 \%$ of patients DAB2 staining was reduced in GC tissues compared to adjacent normal tissues. In order to get an insight into the difference, the DAB2 expression in GC patients with paired adjacent non-tumor tissues and in GC cell lines was investigated using Western blot. As shown (Figure 1B,C), the expression of DAB2 was diminished in two GC tissues. Furthermore, levels of DAB2 expression in all six GC cell lines were decreased obviously in comparison with normal gastric epithelial cells (GES-1), especially in MGC and HGC-27 ones (Figure 1D,E). Therefore, our results indicated that DAB2 expression was decreased in $\mathrm{GC}$ at the protein level.

\section{The aberrant expression of DAB2 influences cell migration in vitro}

To gain further insight into the role of DAB2 in GC, 

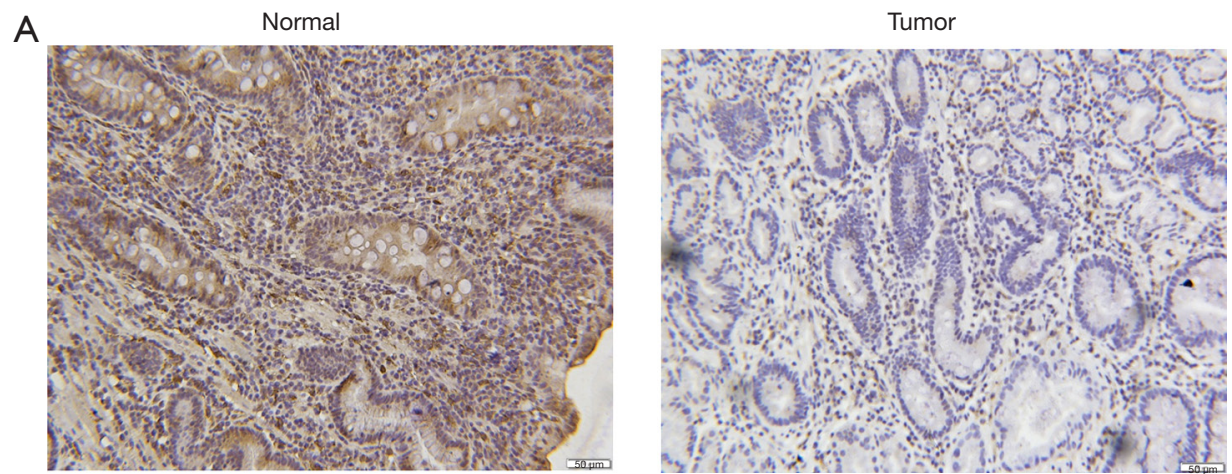

B
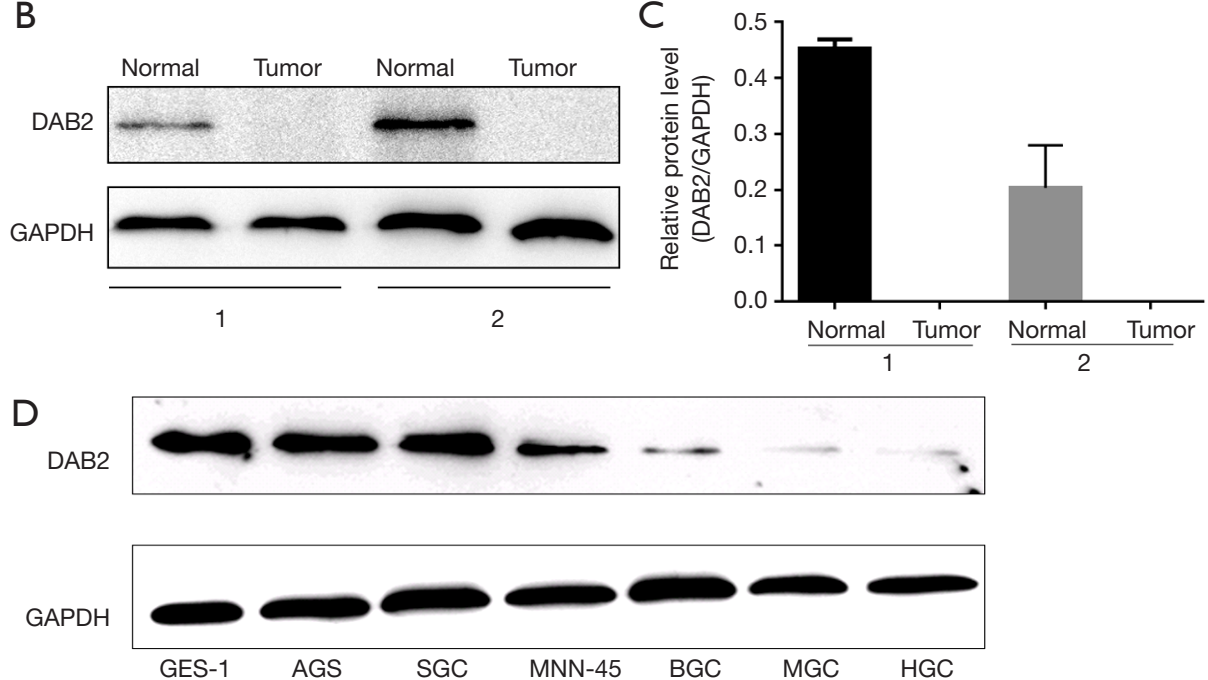

E

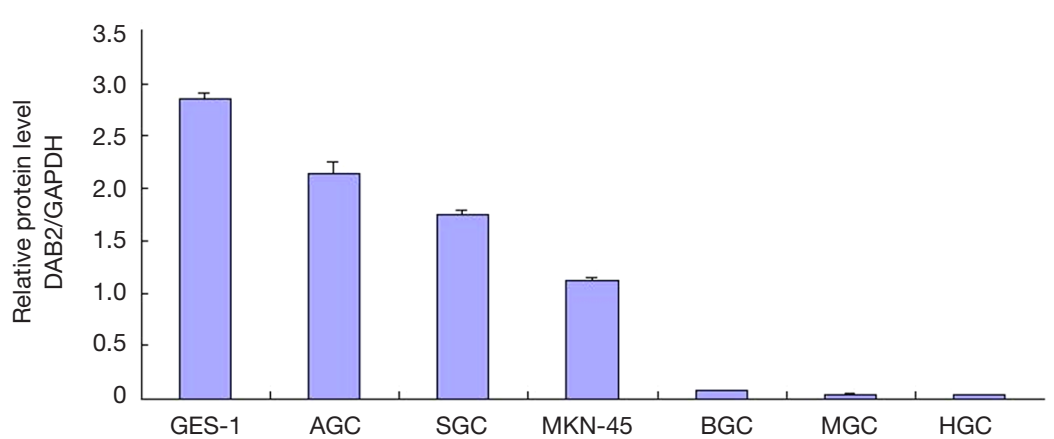

Figure 1 DAB2 expression level was detected in GC and paired adjacent non-tumor tissues, in normal gastric epithelial cells and six GC cell lines by immunohistochemistry and Western blot. (A) Representative immunohistochemical staining of DAB2 was shown in an adjacent normal tissue (left) and GC tissue (right) $(\times 100)$. (B,C) In two pairs of GC patients and adjacent non-tumor tissues, the expression of DAB2 was diminished in GC tissues as detected by Western blot. (D,E) DAB2 protein expression was lower in all six GC cell lines than in GES-1. GC, gastric cancer; DAB2, disabled-2.

SGC cells were transfected with si-DAB2 or si-control, and $\mathrm{DAB} 2$-overexpressed vector or control-vector was transfected into MGC. The expression of DAB2 in SGC and MGC was confirmed by Western blot (Figure $2 A, B, C, D$ ). Then, transwell migration assays were performed. A higher absorbance of si-DAB2 cells compared with si-control cells was observed (Figure 3A,B), and DAB2 overexpression in MGC significantly decreased the number of migratory cells (Figure 3C,D). Meanwhile, Western blot was adopted to detect the expression of a series of molecular markers, such 
A

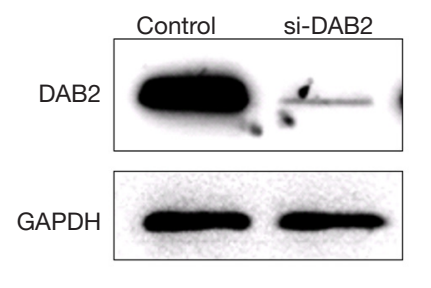

C
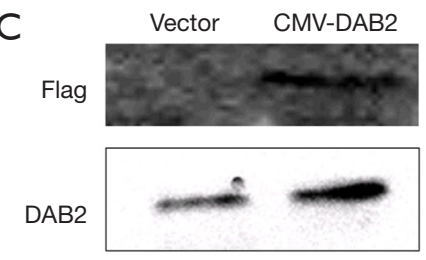

GAPDH

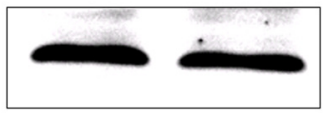

B
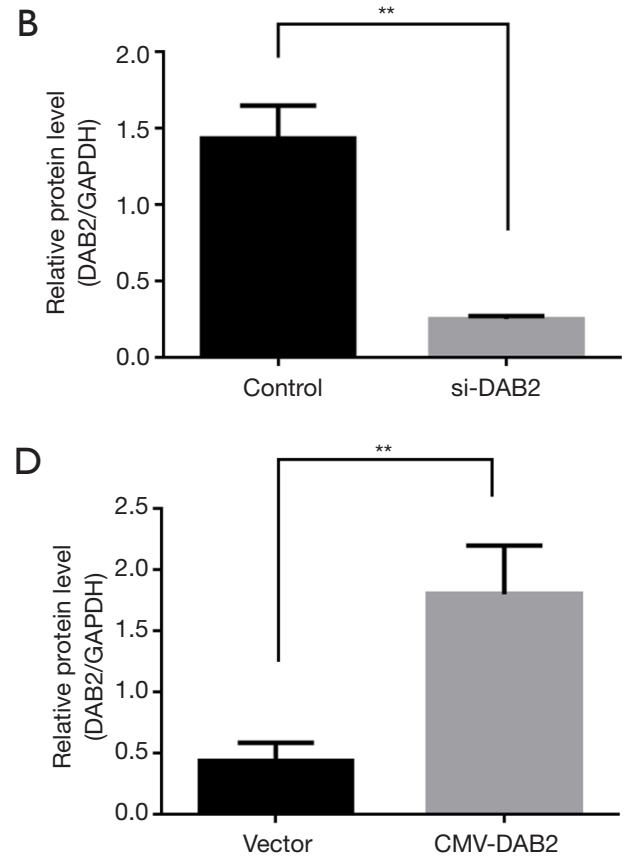

Figure 2 The expression of DAB2 was detected in SGC cells transfected by si-DAB2 and in MGC cells transfected by CMV-MCS-3FLAGSV40-DAB2. (A,B) DAB2 protein expression was obviously decreased in si-DAB2 cells compared with that of the control. (C,D) CMVMCS-3FLAG-SV40-DAB2 could be up-regulated the expression of DAB2 in MGC cells ( $\left.{ }^{* *}, \mathrm{P}<0.01\right)$. DAB2, disabled-2.

as E-cadherin, MMP-2 and MMP-9. As shown in si-DAB2 cells (Figure 3E,F), E-cadherin was lower and the other two (MMP9 and MMP2) were higher than in si-control cells. In contrast, E-cadherin was increased, while MMP-9 and MMP-2 were decreased, in the MGC overexpression group (Figure 3G,H), when compared with control.

\section{$D A B 2$ knockdown results in increased Wnt/B-catenin signaling}

The Wnt/ $\beta$-catenin pathway plays an important role in tumor cell migration and invasion. Thus, the consequences of DAB2 knockdown and overexpression on the influence of the $\mathrm{Wnt} / \beta$-catenin pathway was explored. The expression of PCNA, Myc, CyclinD1, $\beta$-catenin, and phosphorylation of GSK-3 $\beta$ (p-GSK3 $\beta$ ), which are Wnt canonical gene targets, were detected by Western blot (Figure 4). As shown in Figure 4A,B, DAB2 silencing increased the total levels of all five markers compared with the control group. Additionally, to investigate further the inhibitory effects of $\mathrm{DAB} 2$ on $\mathrm{Wnt} / \beta$-catenin signaling, immunofluorescence for $\beta$-catenin was conducted. Just as in Figure $4 E$, in DAB2-si cells, $\beta$-catenin translocated to the nucleus, and expressed ubiquitous amounts in both the nucleus and cytoplasm. These data revealed the same results as those of the Western blot analysis. Moreover, $\beta$-catenin in the control cells was predominantly restricted to the cytoplasm, in contrast to its expression in DAB2-si cells. Otherwise, DAB2 overexpression in MGC cells had not largely changed in comparison with the control cells as detected by immunofluorescence or by Western blot, except for pGSK3 $\beta$, which was dramatically decreased (Figure 4C,D,F). Thus, these results demonstrated that DAB2 silencing upregulated $\mathrm{Wnt} / \beta$-catenin signaling.

\section{DAB2 affects the Hippo-YAP pathway}

The Hippo pathway plays an important role in cancer development (14). To explore whether DAB2 activated the Hippo-YAP pathway, the expression level of Yap, phosphorylated Yap (p-Yap), LATS and phosphorylated LATS (p-LATS) were examined by Western blot. The results showed that the level of p-Yap was obviously decreased in si-DAB2 cells than in control DAB2 cells (Figure $5 A$ ). Consequently, there was an increased in DAB2 over expressed cells (Figure 5B). Moreover, analysis of the ratios of p-Yap/Yap in DAB2 knockdown SGC cells identified a decrease in these phosphorylated proteins 
A

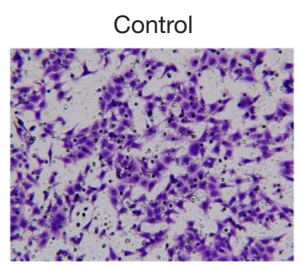

SGC

C

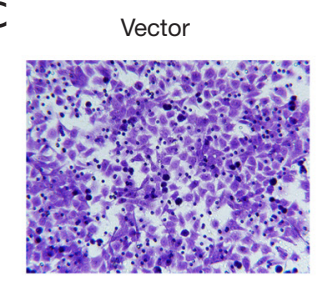

MGC

E

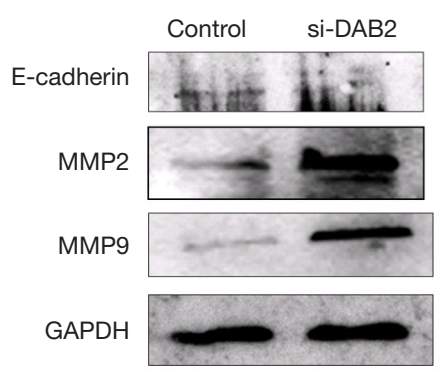

G

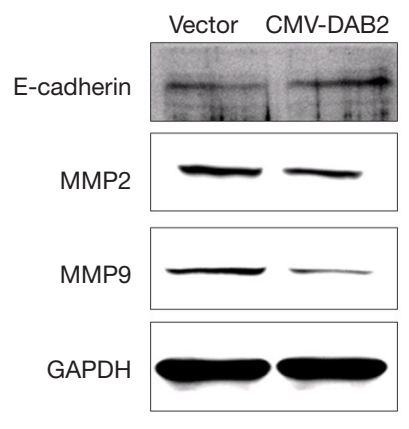

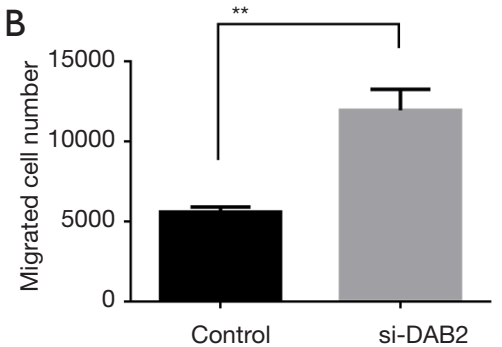

D
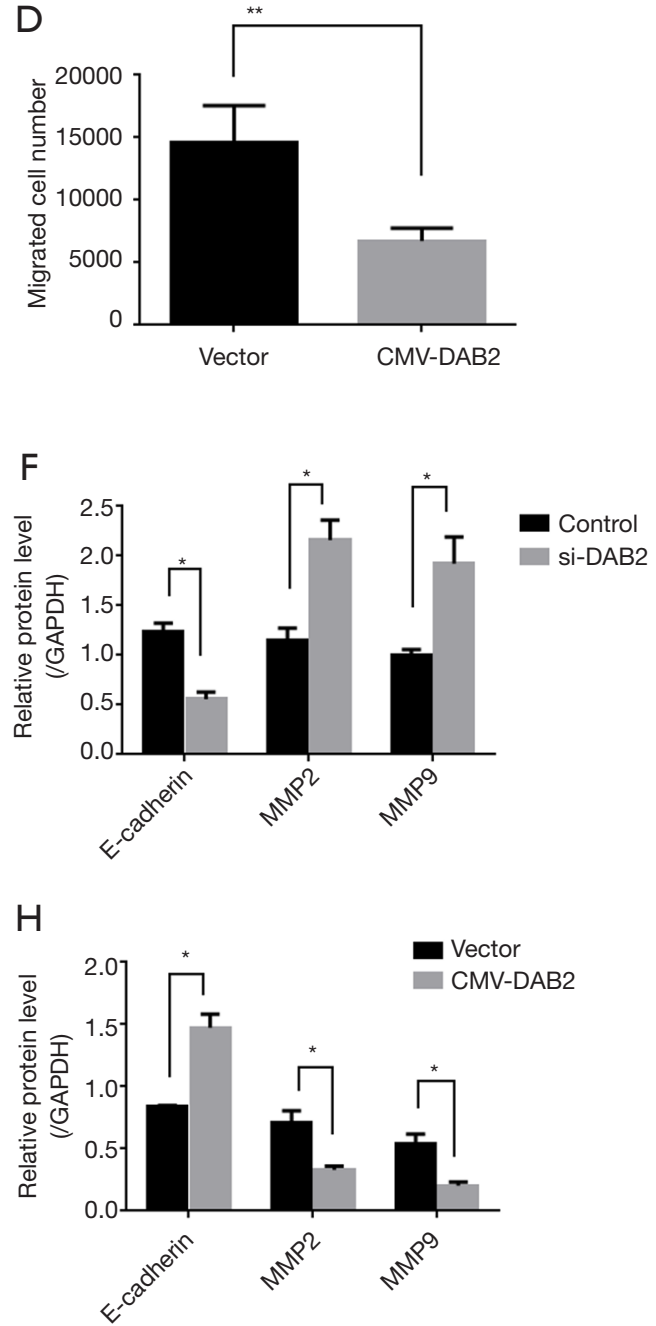

Figure 3 Aberrant DAB2 expression influences cell migration. (A,B) In DAB2-silenced SGC cells, the migratory ability of cell was enhanced as compared with the control SGC cells $(\mathrm{HE}, \times 200)$. (C,D) DAB2 overexpression reduced the migratory ability of MGC cells as compared with the control MGC cells (HE, $\times 200)$. (E-H) Western blot demonstrated MMP-9, as well as MMP-2 and E-cadherin expression levels in si-DAB2 cells and in DAB2-overexpressed DAB2 cells, respectively $\left({ }^{*}, \mathrm{P}<0.05 ;{ }^{* *}, \mathrm{P}<0.01\right)$. DAB2, disabled-2. 


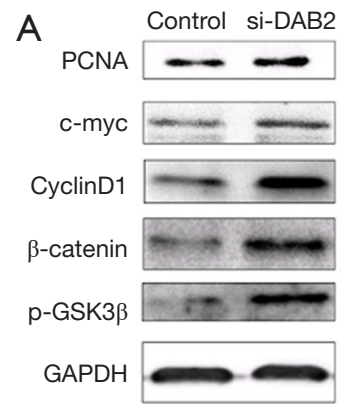

$E$

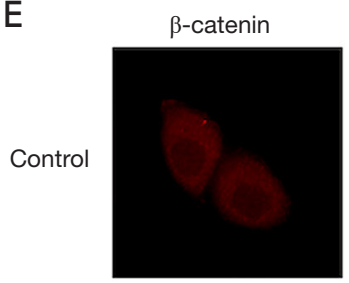

si-DAB2

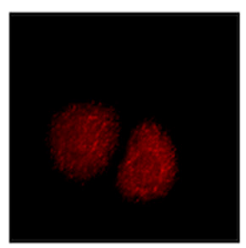

B

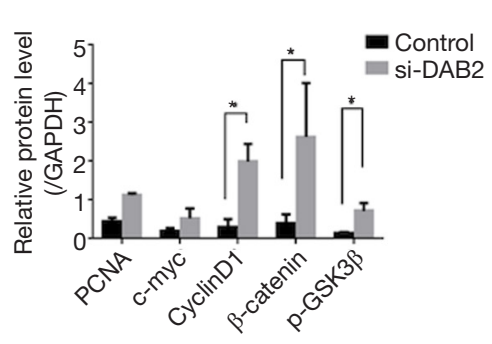

Hoechst
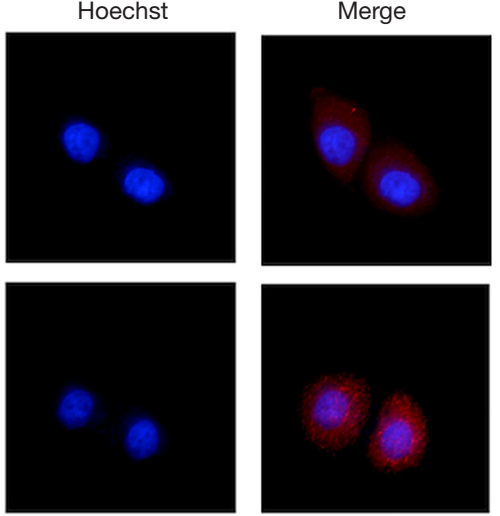

C

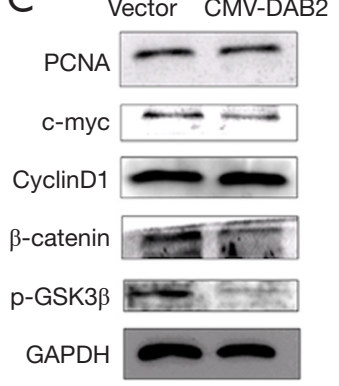

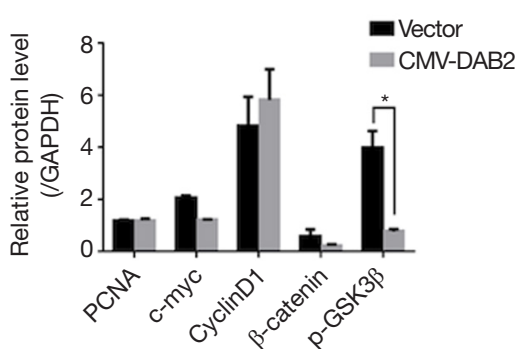

$\mathrm{F}$
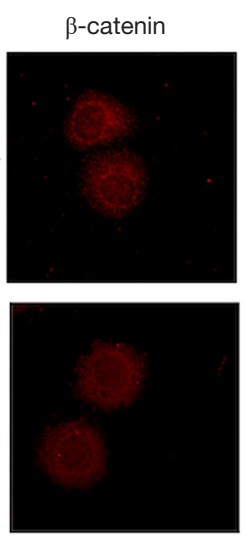

Hoechst
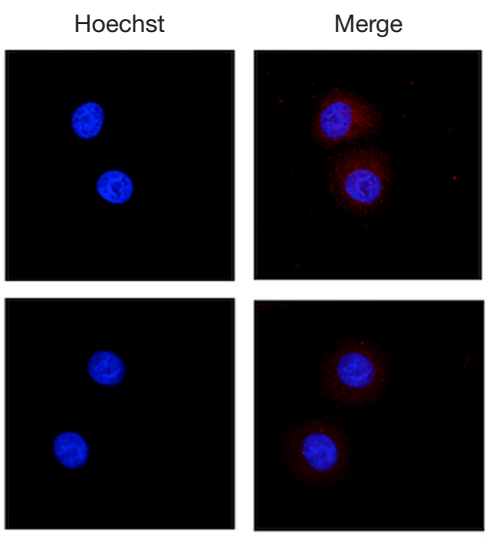

CMV-DAB2

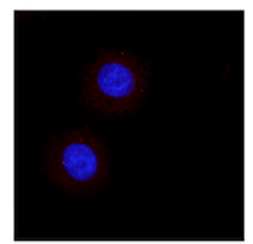

Figure 4 DAB2 expression affected the Wnt/ $\beta$-catenin signaling pathway. (A,B) Western blot analyzed Wnt canonical gene targets in DAB2-knockout cells and showed that CyclinD1, $\beta$-catenin and p-GSK3 $\beta$ were significantly increased in DAB2-silencing cells. (C,D) Western blot analyzed Wnt canonical gene targets in DAB2-overexpressed cells $\left({ }^{*}, \mathrm{P}<0.05\right)$. (E,F) IF assay using anti- $\beta$-catenin (red) antibodies was performed in DAB2-knockout cells and DAB2-overexpressed cells. Hoechst was used to visualized the cell nucleus (blue) (×600). DAB2, disabled-2; IF, immunofluorescence.

(Figure 5C). In addition, overexpression of DAB2 in MGC increased the ratios of $\mathrm{p}$-Yap/Yap. However, in comparison with the control cells, the level of p-LATS and the ratios of p-LATS/LATS had not significant difference either in SGC cells transfected with si-DAB2 or in MGC cells transfected with the DAB2-overexpressed vector (Figure 5B,C). In order to better determine the molecular mechanisms responsible for the effects of DAB2 on the Hippo-YAP pathway, the levels of Yap and p-Yap proteins were analyzed by immunofluorescence. These results were consistent with our previous results in which the level of p-Yap was decreased and Yap translocated to the nucleus in SGC-si cells, and the cells that showed overexpression had no difference compared with the control (Figure 5D,E,F,G). These results suggested that DAB2 was involved in the YAP activation.

\section{Discussion}

Recent evidence has suggested that decrease of DAB2 was observed, and was sustained down the tumorigenic pathway in esophageal squamous cell carcinomas (ESCC) (14). Further study revealed that DAB2 correlated with survival and recurrence (15). Moreover, in lung cancer, the methylation of DAB2 was related to the development of the cancer and might be a predictor of the radio sensitivity of lung cancer $(16,17)$. Here, the expression of DAB2 in different GC cell lines and gastric epithelial cells was firstly investigated through Western blot, and it was found that the level of DAB2 decreased in GC cell lines compared with gastric epithelial cells. Further examination of samples from GC patients with paired adjacent non-tumor tissue by Western blot and immunohistochemical analysis showed that in GC, the expression of DAB2 was lost and $86 \%$ of patients DAB2 staining was reduced in GC tissues compared to adjacent normal tissues. Moreover, transwell analysis was performed, allowing the identification of the migration of affected GC cells. It was found that the down-regulation of DAB2 was able to increase GC cell migration, meanwhile E-cadherin 
A

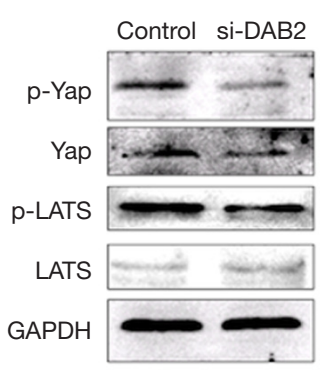

$\mathrm{D}$
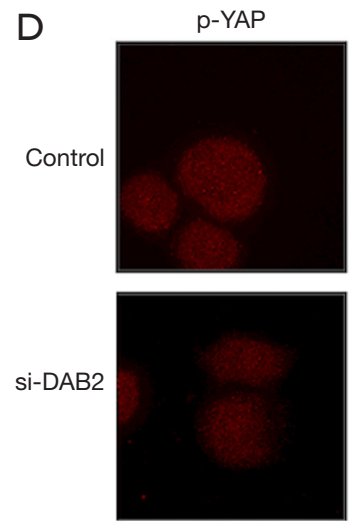

F

Control
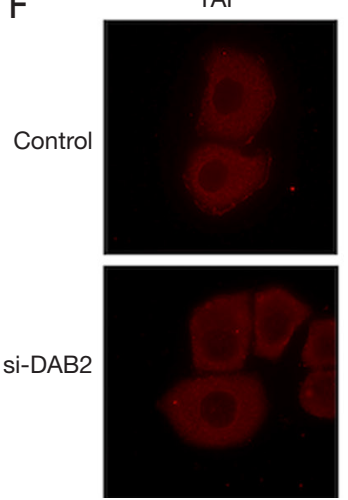

B

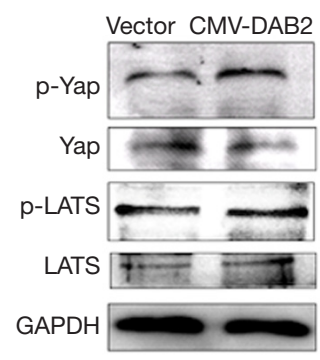

C

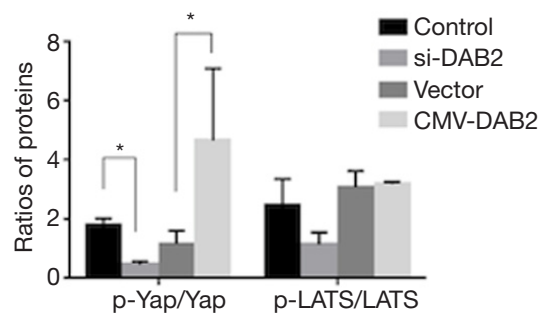

Figure 5 DAB2 regulated the Hippo-YAP pathway. (A,B) The expression level of Yap, p-Yap, LATS1 and p-Lats were examined by Western blot in DAB2-knockout cells and DAB2-overexpressed cells. (C) The ratios of p-Yap/Yap and p-LATS1/LATS1 were analyzed in t DAB2knockout SGC cells and DAB2-overexpressed MGC cells, respectively. (D,E,F,G) IF assay using anti-p-YAP (red) and anti-YAP (red) antibodies was performed in DAB2-knockout cells and DAB2-overexpressed cells, respectively. Hoechst was used to visualized the cell nucleus (blue) (×600).

as epithelial marker was down-regulated, whereas MMP-2 and MMP-9 as mesenchymal markers, were up-regulated. Meanwhile, DAB2 overexpression significantly reduced GC cell migration, and E-cadherin was increased, whereas MMP-2 and MMP-9 were decreased. These findings were consistent with previous works, which has shown that DAB2 regulates and spreads tumor cell migration (18-20).
The $\mathrm{Wnt} / \beta$-catenin signaling pathway plays a crucial role in tumorigenesis (21). Previously, researchers had reported that migration was associated with the regulation of the Wnt signaling pathway $(22,23)$. Moreover, Hocevar found that overexpression of DAB2 suppressed the Wnt signaling pathway through an association with Axin and Dvl-3 (Dishevelled-3) (24), and then Jiang further 
identified DAB2 as the inhibitor of this signal pathway $(10,11,25)$. Thus, what is the relationship between DAB2 and the Wnt signaling pathway in GC? In our study, some important protein targets in the Wnt signal pathway were initially detected by Western blot. In the absence of DAB2, $\beta$-catenin that is an indicator of the Wnt signal pathway activation and $\mathrm{p}-\mathrm{GSK}-3 \beta$ that is one of the core components of this signaling, were highly increased. Additionally, PCNA, c-Myc and CyclinD1 as canonical Wnt signaling direct targets proteins were also increased. During the Wnt signaling, the translocation of $\beta$-catenin from the cytoplasm to the nucleus is a clear indication of the activation of the pathway. So, IF was conducted for $\beta$ catenin. The result showed that $\beta$-catenin translocated to the nucleus in DAB2-silenced cells compared with the control group. Meanwhile, the expression of DAB2 was dramatically increased, and this finding was consistent with our previous result. These results suggested that loss of DAB2 activated the Wnt signal pathway, which mediated cell migration in GC.

The Hippo signaling pathway has been shown to be associated with cancer development $(16,26,27)$ and reported frequently in many cancers, including liver cancer, lung cancer, and ovarian cancer (28-30). Yesassociated protein (YAP), the essential downstream effector of the Hippo signaling pathway, has been observed to be overexpressed in certain cancers (31-33). It was reported that YAP/TAZ, the Hippo transducers, played positive or negative roles in Wnt signaling, and were important components of the $\beta$-catenin destruction complex (34). In our previous results of this study, $\beta$-catenin was increased and translocated from the cytoplasm into the nucleus in DAB2 knockdown cells. Thus, the expression of p-YAP, a result of the activation of the core kinase cassette of the Hippo signaling pathway, and translocation of YAP, were detected. p-Yap was decreased and Yap translocated into the nucleus in DAB2-silenced cells. YAP and $\beta$-catenin in the nuclei interacted with each other to activate the related genes (35). LATS, one of the Hippo core components, and p-LATS were also down-regulated. Furthermore, the ratios of p-Yap/Yap in DAB2 knockdown SGC cells saw a decrease. In addition, overexpression of DAB2 in MGC increased the ratios of p-Yap/Yap. These results suggested that DAB2 was involved in the YAP activation. Thus, our work demonstrated that $\mathrm{p}$-YAP was lost, and $\beta$-catenin accumulated in the nucleus in the Wnt-ON state, an observation that is consistent with what has been reported presently (35).

Overall, our study provides compelling evidence that the lower expression of DAB2 regulates cell migration in GC via interfering with the Wnt and Hippo signaling pathways. This study will be designed to further identify the relationship between DAB2 (as a clinical biomarker) and poor survival, as well as high-risk recurrence in GC.

\section{Acknowledgments}

Funding: This work was supported by Jilin Provincial Education Department (Grant no. JJKH20170861KJ)

\section{Footnote}

Conflicts of Interest: All authors have completed the ICMJE uniform disclosure form (available at http://dx.doi. org/10.21037/tcr.2019.12.96). The authors have no conflicts of interest to declare.

Ethical Statement: The authors are accountable for all aspects of the work in ensuring that questions related to the accuracy or integrity of any part of the work are appropriately investigated and resolved. The study was conducted in accordance with the Declaration of Helsinki (as revised in 2013). Informed consents were obtained from all the participants enrolled, and the study was approved by institutional ethics committee board of Jiangsu University (No. UJS-IACUC-AP-20190307087).

Open Access Statement: This is an Open Access article distributed in accordance with the Creative Commons Attribution-NonCommercial-NoDerivs 4.0 International License (CC BY-NC-ND 4.0), which permits the noncommercial replication and distribution of the article with the strict proviso that no changes or edits are made and the original work is properly cited (including links to both the formal publication through the relevant DOI and the license). See: https://creativecommons.org/licenses/by-nc-nd/4.0/.

\section{References}

1. Ferlay J, Soerjomataram I, Dikshi R, et al. Cancer incidence and mortality worldwide:sources, methods and major patterns in GLOBOCAN 2012. Int J Cancer 2015;136:E359-86.

2. Smyth EC, Cunningham D. Gastric cancer in 
2012:Defining treatment standards and novel insights into disease biology. Nat Rev Clin Oncol 2013;10:73-4.

3. Chu D, Zhu S, Li J, et al. CD147 expression in human gastric cancer is associated with tumor recurrence and prognosis. PLoS One 2014;9:e101027.

4. Morris SM, Cooper JA. Disabled-2 colocalizes with the LDLR in clathrin-coated pits and interacts with AP-2. Traffic 2001;2:111-23.

5. Morris SM, Arden SD, Roberts RC, et al. Myosin VI binds to and localises with Dab2, potentially linking receptormediated endocytosis and the actin cytoskeleton. Traffic 2002;3:331-41.

6. Rosenbauer F, Kallies A, Scheller M, et al. Disabled-2 is transcriptionally regulated by ICSBP and augments macrophage spreading and adhesion. EMBO J 2002;21:211-20.

7. Zhou J, Scholes J, Hsieh JT. Characterization of a novel negative regulator (DOC-2/DAB2) of c-Src in normal prostatic epithelium and cancer. J Biol Chem 2003;278:6936-41.

8. Karam JA, Shariat SF, Huang HY, et al. Decreased DOC2/DAB2 expression in urothelial carcinoma of the bladder. Clin Cancer Res 2007;13:4400-6.

9. Hocevar BA. Loss of Disabled-2 Expression in Pancreatic Cancer Progression. Sci Rep 2019;9:7532.

10. Jiang Y, Luo W, Howe PH. Dab2 stabilizes Axin and attenuates $W n t / b e t a-c a t e n i n$ signaling by preventing protein phosphatase 1 (PP1)-Axin interactions. Oncogene 2009;28:2999-3007.

11. Jiang Y, He X, Howe PH. Disabled-2 (Dab2) inhibits Wnt/beta-catenin signalling by binding LRP6 and promoting its internalization through clathrin. EMBO J 2012;31:2336-49.

12. Kleeff J, Huang Y, Mok SC, et al. Down-regulation of DOC-2 in colorectal cancer points to its role as a tumor suppressor in this malignancy. Dis Colon Rectum 2002;45:1242-8.

13. Anupam K, Tusharkant C, Gupta SD, et al. Loss of disabled-2 expression is an early event in esophageal squamous tumorigenesis. World J Gastroenterol 2006;12:6041-5.

14. Yin M, Zhang L. Hippo signaling: A hub of growth control, tumor suppression and pluripotency maintenance. J Genet Genomics 2011;38:471-81.

15. Wang WL, Chang WL, Yang HB, et al. Low disabled-2 expression promotes tumor progression and determines poor survival and high recurrence of esophageal squamous cell carcinoma. Oncotarget 2016;7:71169-81.

16. Ma S, Zhang WL, Leckey BD Jr, et al. X-ray irradiation induced Disabled-2 genepromoter de-methylation enhancesradiosensitivity of non-small-cell lungcarcinoma cells. J Exp Clin Cancer Res 2018;37:315.

17. Li C, Chen J, Chen T, et al. Aberrant Hypermethylation at Sites -86 to 226 of DAB2 Gene in Non-Small Cell Lung Cancer. Am J Med Sci 2015;349:425-31.

18. Teckchandani A, Toida N, Goodchild J, et al. Quantitative proteomics identifies a Dab2/integrin module regulating cell migration. J Cell Biol 2009;186:99-111.

19. Chetrit D, Ziv N, Ehrlich M. Dab2 regulates clathrin assembly and cell spreading. Biochem J 2009;418:701-15.

20. Xie Y, Zhang Y, Jiang L, et al. Disabled homolog 2 is required for migration and invasion of prostate cancer cells. Front Med 2015;9:312-21.

21. Mi Y, Zhao S, Zhou C, et al. Downregulation of homeobox gene Barx2 increases gastric cancer proliferation and metastasis and predicts poor patient outcomes. Oncotarget 2016;7:60593-60608.

22. Fatima I, El-Ayachi I, Taotao L, et al. The natural compound Jatrophone interferes with Wnt/betacatenin signaling and inhibits proliferation and EMT in human triple-negative breast cancer. PLos One 2017;12:e0189864.

23. Zhang Q, Li Y, Zhao R, et al. The gain-of-function mutation E76K in SHP2 promotes CAC tumorigenesis and induces EMT via the Wnt/beta-catenin signaling pathway. Mol Carcinog 2018;57:619-28.

24. Hocevar BA, Mou F, Rennolds JL, et al. Regulation of the Wnt signaling pathway by disabled-2 (Dab2). EMBO J 2003;22:3084-94.

25. Hofsteen P, Robitaille AM, Chapman DP, et al. Quantitative proteomics identify DAB2 as a cardiac developmental regulator that inhibits WNT/beta-catenin signaling. Proc Natl Acad Sci U S A 2016;113:1002-7.

26. Mo JS, Park HW, Guan KL. The Hippo signaling pathway in stem cell biology and cancer. EMBO Rep 2014;15:642-56.

27. Zhao B, Li L, Lei Q, et al. The Hippo-YAP pathway in organ size control and tumorigenesis: An updated version. Genes Dev 2010;24:862-74.

28. Wang S, Li H, Wang G, et al. Yes-associated protein (YAP) expression is involved in epithelial-mesenchymal transition in hepatocellular carcinoma. Clin Transl Oncol 2016;18:172-7.

29. Zhao B, Ye X, Yu J, et al. TEAD mediates YAP- 
dependent gene induction and growth control. Genes Dev 2008;22:1962-71.

30. Fernandez-L A, Squatrito M, Northcott P, et al. Oncogenic YAP promotes radioresistance and genomic instability in medulloblastoma through IGF2-mediated Akt activation. Oncogene 2012;31:1923-37.

31. Zhou Z, Zhu JS, Gao CP, et al. siRNA targeting YAP gene inhibits gastric carcinoma growth and tumor metastasis in SCID mice. Oncol Lett 2016;11:2806-14.

32. Liu JY, Li YH, Lin HX, et al. Overexpression of YAP 1 contributes to progressive features and poor prognosis of human urothelial carcinoma of the bladder. BMC Cancer

Cite this article as: Wang H, Dong S, Liu Y, Ma F, Fang J, Zhang W, Shao S, Shen H, Jin J. DAB2 suppresses gastric cancer migration by regulating the $\mathrm{Wnt} / \beta$-catenin and HippoYAP signaling pathways. Transl Cancer Res 2020;9(2):11741184. doi: $10.21037 /$ tcr.2019.12.96
2013;13:349.

33. Muramatsu T, Imoto I, Matsui T, et al. YAP is a candidate oncogene for esophageal squamous cell carcinoma. Carcinogenesis 2011;32:389-98.

34. Azzolin L, Panciera T, Soligo S, et al. YAP/TAZ incorporation in the beta-catenin destruction complex orchestrates the Wnt response. Cell 2014;158:157-70.

35. Santucci M, Vignudelli T, Ferrari S, et al. The Hippo Pathway and YAP/TAZ-TEAD Protein-Protein Interaction as Targets for Regenerative Medicine and Cancer Treatment. J Med Chem 2015;58:4857-73. 